\title{
Treatment patterns and health care costs among patients with psoriatic arthritis treated with biologic or targeted synthetic disease-modifying antirheumatic drugs
}

\author{
Mwangi J Murage, PhD, MPH; Nicole Princic, MS; Julie Park, MPH; William N Malatestinic, PharmD, MBA; \\ Baojin Zhu, PhD; Bilal Atiya, PharmD; Scott A Kern, RN, BSN; Keri B Stenger, PharmD; Aubrey Trevelin Sprabery, MD; \\ and Alexis Ogdie, MD
}

\section{What is already known about this subject}

- Multiple biologic and targeted synthetic disease-modifying antirheumatic drug (tsDMARD) treatment options exist for psoriatic arthritis (PsA), and patient adherence, persistence, discontinuation, switching, and health care costs vary among these options.

- Switching or discontinuing biologics can result in higher health care utilization and medical costs.

- Persistent biologic therapy has been associated with superior clinical outcomes compared with interrupted therapy.

\section{What this study adds}

- This study describes a large number of older and more recently approved biologic and tsDMARD treatment patterns and costs among PsA patients.

- Adjustments using published discount factors provide a more realistic estimate of real-world costs for these drugs.

\section{ABSTRACT}

BACKGROUND: Biologic and targeted synthetic disease-modifying antirheumatic drug (tsDMARD) therapies are used in management of psoriatic arthritis (PsA). Although previous studies have demonstrated that rates of adherence, persistence, discontinuation, and switching, as well as health care costs, are variable among treatments, limited published data exist on more recently approved therapies.
OBJECTIVE: To describe adherence, persistence, discontinuation, reinitiation, switching, dosing patterns, and health care costs among PsA patients treated with biologics and tsDMARDs.

METHODS: This was a real-world, retrospective administrative claims study. Adult PsA patients with at least 1 claim for an approved PsA biologic (adalimumab, certolizumab, etanercept, golimumab, infliximab, secukinumab, or ustekinumab) or tsDMARD (apremilast or tofacitinib) between January 1,2015 , and June 30,2019 , were selected

\section{Author affiliations}

Mwangi J Murage, PhD, MPH; William N Malatestinic, PharmD, MBA; Baojin Zhu, PhD; Bilal Atiya, PharmD; Scott A Kern, RN, BSN; Keri B Stenger, PharmD; and Aubrey Trevelin Sprabery, MD, Eli Lilly and Company, Indianapolis, IN. Nicole Princic, MS, and Julie Park, MPH, IBM Watson Health, Cambridge, MA. Alexis Ogdie, MD, Hospital of the University of Pennsylvania, Philadelphia, PA.

AUTHOR CORRESPONDENCE:

Nicole Princic, 781.670.6371;

nprincic@us.ibm.com

J Manag Care Spec Pharm. 2022;28(2):206-17

Copyright $\odot 2022$, Academy of Managed Care Pharmacy. All rights reserved.

from the IBM MarketScan administrative claims databases. The first claim for one of the study treatments determined the index date and drug cohort. Patients were required to be continuously enrolled in their health plans for 12 months before and after the index date and to have at least 1 claim with a diagnosis of PsA in the 12 months before or on the index date. Adherence (measured by proportion of days covered [PDC] and medication possession ratio [MPR]), persistence ( $<60$-day gap in treatment), discontinuation (>90-day gap), reinitiation of index drug, switching, and dosing patterns for each index 
drug were assessed during the 12-month follow-up period. Health care costs were reported per patient per month (PPPM) during the 12-month follow-up and assessed after adjusting PsA treatment costs by the Institute for Clinical and Economic Review discount factors to account for discounts and rebates not usually reflected in claims data and by adherence.

RESULTS: Overall, 6,674 patients met the selection criteria. The top 3 index drugs were adalimumab (37\%), apremilast (26\%), and etanercept (18\%). Over 12 months of follow-up, $31 \%-59 \%$ of patients remained persistent on the index drug, whereas 35\%-56\% discontinued, $13 \%-29 \%$ switched to a different biologic or tsDMARD, and $3 \%-15 \%$ reinitiated the index therapy, depending on the index drug. The mean PDC ranged from 0.51 to 0.69 during the 12 -month follow-up and 0.80 to 0.93 during the follow-up period before discontinuation. Dose values were largely consistent with prescribing information, with the exception of secukinumab. Index drug costs PPPM ranged from $\$ 2,361$ (apremilast) to $\$ 6,528$ for ustekinumab after adjustment for discounts and adherence.

CONCLUSIONS: Results from this real-world analysis suggest that there is substantial variability in persistence, discontinuation, adherence, reinitiating, and switching patterns among the different biologic and tsDMARD treatment options for PsA patients. In addition, this study provides real-world cost data for biologics and tsDMARDs among patients with PsA.

Psoriatic arthritis (PsA) is a chronic inflammatory musculoskeletal disease associated with psoriasis ( $\mathrm{PsO}){ }^{1-3}$ Prognosis varies from a relatively mild form to a severe and destructive polyarticular form with progressive joint deformities. The prevalence of PsA is $0.06 \%$ to $0.25 \%$ of all patients in the United States. ${ }^{3}$ Although the overall prevalence of PsA is low, it is more common among those with $\mathrm{PsO}$, affecting approximately $20 \%$ of patients with PsO. Joint manifestations of PsA may start without the presence of PsO or may develop later in patients with $\mathrm{PsO}$, with an average of 10 years from initial PsO diagnosis to development of PsA. The US economic burden of PsA was estimated from a review of 49 studies from 1998 to 2009 to be as high as $\$ 1.9$ billion annually, ${ }^{4}$ and the cost of psoriatic disease (ie, PsO, PsA, and related comorbid conditions) was reported at $\$ 135$ billion a year in $2013 .{ }^{5}$

More recently, Merola et al showed that patients with a PsA diagnosis between 2009 and 2019 incurred \$29,621 in direct all-cause health care costs per patient per year, compared with $\$ 11,856$ for patients with PsO only and $\$ 7,542$ for patients with neither condition. ${ }^{6}$ In addition, previous research suggests annual health care costs that are $\$ 23,427$ higher for patients with comorbid PsA and PsO (between
2008 and 2013) compared with $\$ 12,523$ among patients with PsO only.

Pharmacologic treatment for PsA includes drugs to manage symptoms (eg, nonsteroidal anti-inflammatory drugs and corticosteroids), conventional disease-modifying antirheumatic drugs (DMARDs), biologics, and targeted synthetic DMARDs (tsDMARDs), ${ }^{7.8}$ Biologics approved by the US Food and Drug Administration for PsA during this study's time period include abatacept, adalimumab, certolizumab, etanercept, golimumab, ixekizumab, infliximab, secukinumab, and ustekinumab; approved tsDMARDs include apremilast and tofacitinib. With the exception of abatacept, golimumab, and tofacitinib, all of these therapies were also indicated for PsO during this study's time period. Treatment selection is affected by disease severity and presence of comorbid conditions such as metabolic disease, cardiovascular conditions, obesity, osteoporosis, and depression/anxiety. ${ }^{8-10}$

Although there are a number of biologic and tsDMARD therapies available for PsA and PsO, adherence to therapy over the first 12 months is low $(0.45-0.67),{ }^{11-13}$ and $35 \%-56 \%$ of patients discontinue therapy at 12 months. ${ }^{13-16}$ This instability in treatment is concerning because continuous therapy with biologics has been associated with superior clinical outcomes compared with interrupted therapy, ${ }^{17,18}$ and switching or discontinuing biologics can result in higher health care utilization and costs. ${ }^{19,20}$

Further research describing treatment patterns and costs are important in understanding how new therapies are used and prescribed. Although there are substantial data on real-world treatment patterns and costs for established PsA therapy options, there are limited published data on more recently approved agents such as abatacept and tofacitinib, both of which were approved for PsA treatment in the United States in 2017.

This retrospective cohort study used real-world data to describe adherence, persistence, discontinuation, restarting, switching, dosing patterns, and health care costs among patients with PsA treated with biologics and tsDMARDs.

\section{Methods}

\section{STUDY DESIGN AND DATA SOURCE}

This retrospective claims study spanned January 1, 2015, through July 31, 2019, and used data from the Commercial Claims and Encounters (Commercial), Medicare Supplemental and Coordination of Benefits (Medicare), and Early View IBM MarketScan databases. The Commercial database contains the inpatient, outpatient, and outpatient prescription drug experience of employees and their 
dependents, covered under a variety of fee-for-service and managed care health plans. The Medicare database contains the same health care data for retirees with Medicare supplemental insurance paid for by employers. The Medicare database captures the Medicare-covered portion of payment (represented as coordination of benefits amount) and the employer-paid portion, so it reflects a patient's full interaction with the health care system. The Early View database contains the same patient data as the Commercial and Medicare databases but captures health care services incurred as late as approximately 45 days before the data release.

All database records were statistically deidentified and certified to be fully compliant with US patient confidentiality requirements set forth in the Health Insurance Portability and Accountability Act of 1996 and therefore exempted from institutional review board approval. All variables used to define study outcomes were obtained using medical codes from International Classification of Diseases, Ninth/ Tenth Revisions, Clinical Modification (ICD-9-CM and ICD10-CM), Healthcare Common Procedure Coding System (HCPCS) codes, and National Drug Code (NDC) numbers.

\section{PATIENT SELECTION}

We identified adults (aged 18+ years) in the MarketScan databases with at least 1 medical or pharmacy claim for a biologic (abatacept, adalimumab, certolizumab, etanercept, golimumab, infliximab, secukinumab, and ustekinumab) or tsDMARD (apremilast and tofacitinib) used to treat PsA between January 1, 2016, and June 30, 2019. The index date was the date of the first qualifying biologic or tsDMARD claim. Ixekizumab, approved for PsA in December 2017, was excluded as an index drug due to limited sample size.

Patients were required to have continuous enrollment with both medical and pharmacy benefits for 12 months before (baseline) and after the index date (follow-up). Patients were required to have at least 1 nondiagnostic claim with a PsA diagnosis (ICD-9-CM code 696.0x or ICD-10-CM code L405.0-L405.9) during the baseline period or on the index date. To ensure that patients were newly initiating the index drug, those with any claims for the index drug during the baseline period were excluded. Patients were segmented into treatment cohorts by index drug.

\section{TREATMENT PATTERNS OUTCOMES}

Treatment persistence, discontinuation, restarting, switching, adherence, and dosing of index PsA treatment were measured over the 12-month follow-up period. For selfadministered oral or subcutaneous (SC) injection drugs, days supply was captured from the NDC record. For clinician-administered SC injection or intravenous (IV) infusion drugs captured by HCPCS codes, days supply was assigned based on prescribing information. Unless otherwise specified, the analysis assumed that overlapping days supply were appended for self-administered drugs and truncated for clinician-administered drugs.

Index treatment was considered persistent if gaps in treatment were less than 60 days. Persistence ended on the day of the last days supply before the first 60-day gap. The percentage of patients who were persistent through the end of the entire post-index period and the number of days to nonpersistence or censoring was calculated. Sensitivity analyses were performed using $45-$ and 90 -day treatment gaps.

Discontinuation was defined as having a gap in therapy of at least 90 days, and the discontinuation date was the last days supply before the 90-day gap. The percentage of patients who discontinued and the number of days from the index date to discontinuation were calculated. Patients with at least 1 claim for their index medication after discontinuing index therapy were defined as restarting therapy. The percentage of patients who restarted index therapy and the number of days from discontinuation to restart were calculated.

Switching was defined as having a new biologic or tsDMARD from index therapy, and although the new therapy may have started while the patient was still receiving index therapy, it only qualified as a switch if there was at least 30 days supply of the new drug as monotherapy after discontinuation of index therapy. Although not included as an index drug, ixekizumab was included as a possible new drug in the switching analysis, since it was available as a treatment option during the follow-up period.

If the new treatment had less than 30 days overlapping with the index treatment, the switch date was set as the date of the first claim of the new therapy. If new treatment had at least 30 days overlapping with the index treatment, the switching date was set as the first drug supply date after the overlapping period. The percentage of patients who switched, the drugs to which they switched, and the number of days from index date to switch date were calculated.

The primary measure of adherence was the proportion of days covered (PDC), defined as the number of days covered by the index drug divided by the time period of interest, which was measured during the full 12-month follow-up period and also during the time before discontinuation. Adherence to the index drug before discontinuation was calculated to examine adherence during a period of active treatment. For calculation of the PDC, overlapping days supply between consecutive fills were truncated for both self- and clinician-administered drugs. The secondary 
measure of adherence was the medication possession ratio (MPR), which was calculated similarly to the PDC except that overlapping days supply between consecutive fills were appended for self-administered drugs and truncated for clinician-administered drugs.

Dosing was calculated for self-administered drugs by multiplying the drug strength by the quantity and dividing by the days of supply for each claim. For clinician-administered drugs, dosing was estimated based on prescribing guidelines. Dosing of index drugs administered through IV infusion or drugs where dose was adjusted according to a patient's weight (ie, infliximab, golimumab-IV, and ustekinumab) were not reported because weight-based dosing did not allow for accurate comparison with prescribing information due to the lack of information on patient weight in the claims data.

For etanercept, adalimumab, golimumab-SC, apremilast, and tofacitinib, dosing was calculated from the index date until the earlier of treatment discontinuation or end of the 12-month follow-up period among patients with at least 1 valid claim. Renal/hepatic function was not captured and thus was not accounted for in dosing analyses for tsDMARDs. For secukinumab and certolizumab, which have an induction and a maintenance phase of treatment administration, patients were required to have at least 1 valid claim in the induction and maintenance phases to be eligible for dosing analysis, and dosing results were reported separately for each phase: the first month after index (induction) and from the first claim after the induction window until the earlier of discontinuation or end of the 12-month follow-up period (maintenance).

The calculated dose for each index drug was compared with the expected dose per the prescribing information for each drug. For patients on secukinumab, dosing analysis was reported separately for patients with and without comorbid PsO due to differences in the prescribing recommendations.

Only valid drug claims were used in the dosing analysis. Claims were considered invalid if quantity, days supply, or pay were less than or equal to zero or if the dosing value was greater than $200 \%$ of the maximum dose or less than $25 \%$ of minimum dose based on prescribing information. Less than $1 \%$ of claims were dropped due to a zero value, and overall, roughly $3 \%$ of patients in any index drug cohort were dropped from the dosing analysis due to invalid drug claims.

\section{HEALTH CARE COST OUTCOMES}

All-cause and PsA-related health care costs over the 12 months of follow-up were measured per patient per month (PPPM) for each index drug and adjusted for inflation to 2019 US dollars using the medical care component of the Consumer Price Index. ${ }^{21}$ Costs were reported overall, by out-of-pocket and plan-paid costs, and by type of service, including inpatient services, outpatient services, and outpatient pharmacy. Health care costs were based on paid amounts of adjudicated claims, including insurer and health plan payments, and the patient-paid portion (ie, copayment, deductible, and coinsurance).

PsA-related costs included inpatient claims with a primary diagnosis for PsA, outpatient medical claims with any diagnosis for PsA, and outpatient claims (medical and pharmacy) for PsA-related treatment. PsA-related treatments consisted of biologic DMARDs, tsDMARDs, and other conventional synthetic DMARDs (ie, azathioprine, methotrexate, leflunomide, and sulfasalazine). Index drug costs and other PsA-related drug costs were reported separately.

To account for discounts, such as rebates, patient assistance programs, and commission to wholesalers that are not consistently captured in claims, Institute for Clinical and Economic Review (ICER) discount rates, based on net price divided by wholesale acquisition costs, were applied to index drug costs where applicable. ${ }^{22}$ Because ICER rates for PsA were not available, the most recent PsO ICER rates were used. Costs could not be adjusted for abatacept, golimumab, and tofacitinib because these drugs are not approved to treat $\mathrm{PsO}$ and therefore were not included in the ICER report. Drug costs may also be affected by adherence (ie, higher adherence means more consumption of drugs and therefore higher costs), so index drug costs were additionally adjusted by adherence rates.

\section{OTHER VARIABLES}

Demographic characteristics (age, sex, payer [commercial or Medicare], and index year) were measured on the index date. The Deyo-Charlson Comorbidity Index score was calculated using claims data from the baseline period. The number and percentage of patients with comorbid $\mathrm{PsO}$ and other conditions (hypertension, hyperlipidemia, obesity osteoarthritis, and other autoimmune disorders) were measured during the baseline period.

Receipt of PsA-related treatment during the baseline period was recorded and included the use of biologics or tsDMARDs, the number of unique biologics or tsDMARDs, and the use of conventional synthetic DMARDs (ie, azathioprine, cyclosporine, methotrexate, leflunomide, and sulfasalazine). The use of systemic corticosteroids or conventional synthetic DMARDs concomitant with the index therapy was also recorded. 


\section{TABLE 1 Patient Characteristics and PsA-Related Treatment During the 12 Months Before Index}

\begin{tabular}{|c|c|c|c|c|c|c|c|c|c|c|}
\hline & $\begin{array}{c}\text { ADA } \\
n=2,481\end{array}$ & $\begin{array}{l}\text { CERT } \\
n=71\end{array}$ & $\begin{array}{c}\text { ETA } \\
n=1,200\end{array}$ & $\begin{array}{c}\text { GOL-IV } \\
n=54\end{array}$ & $\begin{array}{c}\text { GOL-SC } \\
n=59\end{array}$ & $\begin{array}{c}\text { INF } \\
n=203\end{array}$ & $\begin{array}{c}\text { SEC } \\
\mathrm{n}=367\end{array}$ & $\begin{array}{c}\text { UST } \\
n=362\end{array}$ & $\begin{array}{c}\text { APR } \\
\mathrm{n}=1,705\end{array}$ & $\begin{array}{c}\text { TOF } \\
n=37\end{array}$ \\
\hline Age, mean (SD) & $\begin{array}{c}48.3 \\
(11.2)\end{array}$ & $\begin{array}{c}53.9 \\
(14.0)\end{array}$ & $\begin{array}{c}49.9 \\
(10.8)\end{array}$ & $\begin{array}{c}51.4 \\
(12.3)\end{array}$ & $\begin{array}{l}50.0 \\
(9.5)\end{array}$ & $\begin{array}{c}52.1 \\
(12.9)\end{array}$ & $\begin{array}{c}49.9 \\
(10.3)\end{array}$ & $\begin{array}{l}50.0 \\
(12.6)\end{array}$ & $\begin{array}{c}52.5 \\
(11.2)\end{array}$ & $\begin{array}{l}58.8 \\
(9.0)\end{array}$ \\
\hline Male, n (\%) & $\begin{array}{l}1,099 \\
(44.3)\end{array}$ & $\begin{array}{c}46 \\
(26.9)\end{array}$ & $\begin{array}{c}502 \\
(41.8)\end{array}$ & $\begin{array}{c}22 \\
(40.7)\end{array}$ & $\begin{array}{c}19 \\
(32.2)\end{array}$ & $\begin{array}{c}66 \\
(32.5)\end{array}$ & $\begin{array}{c}158 \\
(43.1)\end{array}$ & $\begin{array}{c}158 \\
(43.7)\end{array}$ & $\begin{array}{c}656 \\
(38.5)\end{array}$ & $\begin{array}{c}11 \\
(29.7)\end{array}$ \\
\hline Commercial payer, $\mathbf{n}(\%)$ & $\begin{array}{l}2,381 \\
(96.0) \\
\end{array}$ & $\begin{array}{c}137 \\
(80.1)\end{array}$ & $\begin{array}{l}1,135 \\
(94.6) \\
\end{array}$ & $\begin{array}{c}48 \\
(88.9) \\
\end{array}$ & $\begin{array}{c}58 \\
(98.3) \\
\end{array}$ & $\begin{array}{c}168 \\
(82.8)\end{array}$ & $\begin{array}{c}350 \\
(95.4) \\
\end{array}$ & $\begin{array}{c}324 \\
(89.5) \\
\end{array}$ & $\begin{array}{l}1,541 \\
(90.4) \\
\end{array}$ & $\begin{array}{c}32 \\
(86.5) \\
\end{array}$ \\
\hline $\begin{array}{l}\text { Deyo Charlson Comorbidity } \\
\text { Index, mean (SD) }\end{array}$ & $\begin{array}{c}0.6 \\
(1.1)\end{array}$ & $\begin{array}{c}0.9 \\
(1.3)\end{array}$ & $\begin{array}{c}0.7 \\
(1.1)\end{array}$ & $\begin{array}{l}1.1 \\
(1.3)\end{array}$ & $\begin{array}{c}0.7 \\
(0.9)\end{array}$ & $\begin{array}{c}1.0 \\
(1.6)\end{array}$ & $\begin{array}{c}0.8 \\
(1.3)\end{array}$ & $\begin{array}{c}0.7 \\
(1.1)\end{array}$ & $\begin{array}{c}1.0 \\
(1.6)\end{array}$ & $\begin{array}{c}1.2 \\
(1.4)\end{array}$ \\
\hline \multicolumn{11}{|l|}{ Index year, n (\%) } \\
\hline 2016 & $\begin{array}{l}1,108 \\
(44.7)\end{array}$ & $\begin{array}{c}105 \\
(61.4)\end{array}$ & $\begin{array}{c}648 \\
(54.0) \\
\end{array}$ & $\begin{array}{c}15 \\
(27.8)\end{array}$ & $\begin{array}{c}46 \\
(78.0)\end{array}$ & $\begin{array}{c}120 \\
(59.1)\end{array}$ & $\begin{array}{c}179 \\
(48.8)\end{array}$ & $\begin{array}{c}212 \\
(58.6)\end{array}$ & $\begin{array}{c}813 \\
(47.7)\end{array}$ & $\begin{array}{c}14 \\
(37.8)\end{array}$ \\
\hline 2017 & $\begin{array}{c}897 \\
(36.2)\end{array}$ & $\begin{array}{c}39 \\
(22.8)\end{array}$ & $\begin{array}{c}370 \\
(30.8)\end{array}$ & $\begin{array}{c}8 \\
(14.8) \\
\end{array}$ & $\begin{array}{c}11 \\
(18.6)\end{array}$ & $\begin{array}{c}48 \\
(23.7)\end{array}$ & $\begin{array}{c}85 \\
(23.2)\end{array}$ & $\begin{array}{c}104 \\
(28.7)\end{array}$ & $\begin{array}{c}476 \\
(27.9) \\
\end{array}$ & $\begin{array}{c}8 \\
(21.6) \\
\end{array}$ \\
\hline 2018 & $\begin{array}{c}476 \\
(19.2) \\
\end{array}$ & $\begin{array}{c}27 \\
(15.8) \\
\end{array}$ & $\begin{array}{c}182 \\
(15.2) \\
\end{array}$ & $\begin{array}{c}31 \\
(57.4) \\
\end{array}$ & $\begin{array}{c}2 \\
(3.4) \\
\end{array}$ & $\begin{array}{c}35 \\
(17.2) \\
\end{array}$ & $\begin{array}{c}103 \\
(28.1) \\
\end{array}$ & $\begin{array}{c}46 \\
(12.7) \\
\end{array}$ & $\begin{array}{c}416 \\
(24.4) \\
\end{array}$ & $\begin{array}{c}15 \\
(40.5) \\
\end{array}$ \\
\hline \multicolumn{11}{|l|}{ Comorbid conditions, $\mathrm{n}(\%)$} \\
\hline Psoriasis & $\begin{array}{l}1,669 \\
(67.3)\end{array}$ & $\begin{array}{c}92 \\
(53.8)\end{array}$ & $\begin{array}{c}726 \\
(60.5)\end{array}$ & $\begin{array}{c}26 \\
(48.2)\end{array}$ & $\begin{array}{c}32 \\
(54.2)\end{array}$ & $\begin{array}{c}121 \\
(59.6)\end{array}$ & $\begin{array}{c}280 \\
(76.3)\end{array}$ & $\begin{array}{c}283 \\
(78.2)\end{array}$ & $\begin{array}{l}1,236 \\
(72.5)\end{array}$ & $\begin{array}{c}17 \\
(46.0)\end{array}$ \\
\hline Hypertension & $\begin{array}{c}937 \\
(37.8)\end{array}$ & $\begin{array}{c}81 \\
(47.4)\end{array}$ & $\begin{array}{c}468 \\
(39.0)\end{array}$ & $\begin{array}{c}26 \\
(48.2)\end{array}$ & $\begin{array}{c}22 \\
(37.3)\end{array}$ & $\begin{array}{c}83 \\
(40.9)\end{array}$ & $\begin{array}{c}145 \\
(39.5)\end{array}$ & $\begin{array}{c}139 \\
(38.4)\end{array}$ & $\begin{array}{c}734 \\
(43.1)\end{array}$ & $\begin{array}{c}18 \\
(48.7)\end{array}$ \\
\hline Hyperlipidemia & $\begin{array}{c}751 \\
(30.3)\end{array}$ & $\begin{array}{c}66 \\
(38.6)\end{array}$ & $\begin{array}{c}379 \\
(31.6)\end{array}$ & $\begin{array}{c}18 \\
(33.3)\end{array}$ & $\begin{array}{c}15 \\
(25.4)\end{array}$ & $\begin{array}{c}72 \\
(35.5)\end{array}$ & $\begin{array}{c}105 \\
(28.6)\end{array}$ & $\begin{array}{c}137 \\
(37.9)\end{array}$ & $\begin{array}{c}621 \\
(36.4)\end{array}$ & $\begin{array}{c}12 \\
(32.4)\end{array}$ \\
\hline Obesity & $\begin{array}{c}695 \\
(28.0)\end{array}$ & $\begin{array}{c}46 \\
(26.9)\end{array}$ & $\begin{array}{c}315 \\
(26.3)\end{array}$ & $\begin{array}{c}19 \\
(35.2)\end{array}$ & $\begin{array}{c}15 \\
(25.4)\end{array}$ & $\begin{array}{c}55 \\
(27.1)\end{array}$ & $\begin{array}{c}111 \\
(30.3)\end{array}$ & $\begin{array}{c}81 \\
(22.4)\end{array}$ & $\begin{array}{c}501 \\
(29.4)\end{array}$ & $\begin{array}{c}10 \\
(27.0)\end{array}$ \\
\hline Osteoarthritis & $\begin{array}{c}800 \\
(32.3)\end{array}$ & $\begin{array}{c}73 \\
(42.7)\end{array}$ & $\begin{array}{c}407 \\
(33.9)\end{array}$ & $\begin{array}{c}17 \\
(31.5)\end{array}$ & $\begin{array}{c}17 \\
(28.8)\end{array}$ & $\begin{array}{c}74 \\
(36.5)\end{array}$ & $\begin{array}{c}99 \\
(27.0)\end{array}$ & $\begin{array}{c}98 \\
(27.1)\end{array}$ & $\begin{array}{c}551 \\
(32.3)\end{array}$ & $\begin{array}{c}16 \\
(43.2)\end{array}$ \\
\hline Other autoimmune disorders ${ }^{a}$ & $\begin{array}{l}132 \\
(5.3)\end{array}$ & $\begin{array}{c}13 \\
(7.6)\end{array}$ & $\begin{array}{c}78 \\
(6.5)\end{array}$ & $\begin{array}{c}4 \\
(7.4)\end{array}$ & $\begin{array}{c}2 \\
(3.4)\end{array}$ & $\begin{array}{c}10 \\
(4.9)\end{array}$ & $\begin{array}{c}36 \\
(9.8)\end{array}$ & $\begin{array}{c}30 \\
(8.3)\end{array}$ & $\begin{array}{l}158 \\
(9.3)\end{array}$ & $\begin{array}{c}6 \\
(16.2)\end{array}$ \\
\hline \multicolumn{11}{|c|}{ Biologic or tsDMARD pre-index use, $n(\%)$} \\
\hline $\begin{array}{l}\text { Treatment naive } \\
\text { (no prior use) }\end{array}$ & $\begin{array}{l}2,356 \\
(95.0)\end{array}$ & $\begin{array}{l}119 \\
(69.6)\end{array}$ & $\begin{array}{l}1,095 \\
(91.3)\end{array}$ & $\begin{array}{c}45 \\
(83.3)\end{array}$ & $\begin{array}{c}37 \\
(62.7)\end{array}$ & $\begin{array}{c}167 \\
(82.3)\end{array}$ & $\begin{array}{c}258 \\
(70.3)\end{array}$ & $\begin{array}{c}268 \\
(74.0)\end{array}$ & $\begin{array}{l}1,526 \\
(89.5)\end{array}$ & $\begin{array}{c}31 \\
(83.8)\end{array}$ \\
\hline $\begin{array}{l}\text { Treatment experienced } \\
\text { (with prior use) }\end{array}$ & $\begin{array}{l}125 \\
(5.0)\end{array}$ & $\begin{array}{c}52 \\
(30.4)\end{array}$ & $\begin{array}{l}105 \\
(8.8)\end{array}$ & $\begin{array}{c}9 \\
(16.7)\end{array}$ & $\begin{array}{c}22 \\
(37.3)\end{array}$ & $\begin{array}{c}36 \\
(17.7)\end{array}$ & $\begin{array}{l}109 \\
(29.7)\end{array}$ & $\begin{array}{c}94 \\
(26.0)\end{array}$ & $\begin{array}{c}179 \\
(10.5)\end{array}$ & $\begin{array}{c}6 \\
(16.2)\end{array}$ \\
\hline $\begin{array}{l}\text { Number of biologic or } \\
\text { tsDMARDs used, mean (SD) }\end{array}$ & $\begin{array}{l}1.1 \\
(0.3)\end{array}$ & $\begin{array}{l}1.2 \\
(0.4) \\
\end{array}$ & $\begin{array}{l}1.2 \\
(0.4) \\
\end{array}$ & $\begin{array}{l}1.4 \\
(0.7)\end{array}$ & $\begin{array}{l}1.3 \\
(0.6)\end{array}$ & $\begin{array}{l}1.2 \\
(0.4)\end{array}$ & $\begin{array}{l}1.3 \\
(0.5)\end{array}$ & $\begin{array}{l}1.3 \\
(0.5) \\
\end{array}$ & $\begin{array}{l}1.1 \\
(0.3)\end{array}$ & $\begin{array}{l}1.3 \\
(0.5)\end{array}$ \\
\hline
\end{tabular}

\section{STATISTICAL ANALYSES}

Continuous variables were presented as means and SDs. Categorical variables were presented as counts and percentages. All data analyses were conducted using WPS version 4.1 (World Programming).

\section{Results}

\section{PATIENT CHARACTERISTICS}

A total of 6,674 patients with PsA newly initiating a biologic or tsDMARD were eligible for study inclusion (Supplementary 


\begin{tabular}{|c|c|c|c|c|c|c|c|c|c|c|}
\hline & $\begin{array}{c}\text { ADA } \\
n=2,481\end{array}$ & $\begin{array}{l}\text { CERT } \\
n=71\end{array}$ & $\begin{array}{c}\text { ETA } \\
n=1,200\end{array}$ & $\begin{array}{c}\text { GOL-IV } \\
n=54\end{array}$ & $\begin{array}{c}\text { GOL-SC } \\
n=59\end{array}$ & $\begin{array}{c}\text { INF } \\
n=203\end{array}$ & $\begin{array}{c}\text { SEC } \\
n=367\end{array}$ & $\begin{array}{c}\text { UST } \\
n=362\end{array}$ & $\begin{array}{c}\text { APR } \\
n=1,705\end{array}$ & $\begin{array}{c}\text { TOF } \\
\mathbf{n}=37\end{array}$ \\
\hline \multicolumn{11}{|c|}{ ther pre-index PsA treatments, $\mathrm{n}(\%)$} \\
\hline $\begin{array}{l}\text { Corticosteroids } \\
\text { (oral or injectable) }\end{array}$ & $\begin{array}{l}1,573 \\
(63.4)\end{array}$ & $\begin{array}{c}121 \\
(70.8)\end{array}$ & $\begin{array}{c}796 \\
(66.3)\end{array}$ & $\begin{array}{c}42 \\
(77.8)\end{array}$ & $\begin{array}{c}41 \\
(69.5)\end{array}$ & $\begin{array}{c}151 \\
(74.4)\end{array}$ & $\begin{array}{c}229 \\
(62.4)\end{array}$ & $\begin{array}{c}210 \\
(58.0)\end{array}$ & $\begin{array}{l}1,059 \\
(62.1)\end{array}$ & $\begin{array}{c}30 \\
(81.1)\end{array}$ \\
\hline Methotrexate & $\begin{array}{l}1,100 \\
(44.3)\end{array}$ & $\begin{array}{c}71 \\
(41.5)\end{array}$ & $\begin{array}{c}490 \\
(40.8)\end{array}$ & $\begin{array}{c}23 \\
(42.6)\end{array}$ & $\begin{array}{c}21 \\
(35.6)\end{array}$ & $\begin{array}{c}99 \\
(48.8)\end{array}$ & $\begin{array}{c}116 \\
(31.6)\end{array}$ & $\begin{array}{c}89 \\
(24.6)\end{array}$ & $\begin{array}{c}471 \\
(27.6)\end{array}$ & $\begin{array}{c}18 \\
(48.7)\end{array}$ \\
\hline -eflunomide & $\begin{array}{l}107 \\
(4.3)\end{array}$ & $\begin{array}{l}15 \\
(8.8)\end{array}$ & $\begin{array}{c}52 \\
(4.3)\end{array}$ & $\begin{array}{c}3 \\
(5.6)\end{array}$ & $\begin{array}{c}7 \\
(11.9)\end{array}$ & $\begin{array}{l}15 \\
(7.4)\end{array}$ & $\begin{array}{l}15 \\
(4.1)\end{array}$ & $\begin{array}{c}14 \\
(3.9)\end{array}$ & $\begin{array}{c}64 \\
(3.8)\end{array}$ & $\begin{array}{c}6 \\
(16.2)\end{array}$ \\
\hline Sulfasalazine & $\begin{array}{l}203 \\
(8.2)\end{array}$ & $\begin{array}{c}12 \\
(7.0)\end{array}$ & $\begin{array}{c}126 \\
(10.5)\end{array}$ & $\begin{array}{c}4 \\
(7.4)\end{array}$ & $\begin{array}{c}6 \\
(10.2)\end{array}$ & $\begin{array}{c}23 \\
(11.3)\end{array}$ & $\begin{array}{c}25 \\
(6.8)\end{array}$ & $\begin{array}{c}22 \\
(6.1)\end{array}$ & $\begin{array}{l}146 \\
(8.6)\end{array}$ & $\begin{array}{c}4 \\
(10.8)\end{array}$ \\
\hline Cyclosporine & $\begin{array}{l}11 \\
(0.4)\end{array}$ & $\begin{array}{c}1 \\
(0.6)\end{array}$ & $\begin{array}{c}7 \\
(0.6)\end{array}$ & $\begin{array}{c}0 \\
(0.0)\end{array}$ & $\begin{array}{c}0 \\
(0.0)\end{array}$ & $\begin{array}{c}1 \\
(0.5)\end{array}$ & $\begin{array}{c}8 \\
(2.2)\end{array}$ & $\begin{array}{c}4 \\
(1.1)\end{array}$ & $\begin{array}{c}5 \\
(0.3)\end{array}$ & $\begin{array}{c}0 \\
(0.0)\end{array}$ \\
\hline Azathioprine & $\begin{array}{l}15 \\
(0.6)\end{array}$ & $\begin{array}{c}2 \\
(1.2)\end{array}$ & $\begin{array}{c}7 \\
(0.6)\end{array}$ & $\begin{array}{c}0 \\
(0.0)\end{array}$ & $\begin{array}{c}1 \\
(1.7)\end{array}$ & $\begin{array}{c}3 \\
(1.5)\end{array}$ & $\begin{array}{c}1 \\
(0.3)\end{array}$ & $\begin{array}{c}2 \\
(0.6)\end{array}$ & $\begin{array}{c}7 \\
(0.4)\end{array}$ & $\begin{array}{c}0 \\
(0.0)\end{array}$ \\
\hline
\end{tabular}

${ }^{a}$ Other autoimmune disorders include Addisons's disease, alopecia areata, celiac disease, chronic glomerulonephritis, chronic urtcaria, giant cell arteritis, pulmonary fibrosis, Sjogren's syndrome, systemic lupus eruthematosus, systemic sclerosis, and vitiligo.

$A D A=$ adalimumab; $A P R=$ apremilast; $C E R T=$ certolizumab; $E T A=$ etanercept; $G O L-I V=$ golimumab intravenous; $G O L-S C=$ golimumab subcutaneous; $I N F=$ infliximab; PSA = psoriatic arthritis; $S E C=$ secukinumab; TOF = tofacitinib; tsDMARD = targeted synthetic disease-modifying antirheumatic drug; UST =ustekinumab.

Figure 1, available in online article). Adalimumab was the most common index drug (37.2\%), followed by apremilast (25.5\%) and etanercept (18.0\%). Patients who indexed on abatacept were not included in the final analysis due to sample size restrictions (ie, less than 30 patients) on reporting MarketScan data.

Across index drugs, patients had a mean age of 48.3 to 58.8 years, and over $50 \%$ were female (Table 1 ). The percentage of PsA patients with comorbid $\mathrm{PsO}$ ranged from $46.0 \%$ of the tofacitinib cohort to $78.2 \%$ of the ustekinumab cohort. Over $60 \%$ of patients in each index drug cohort were naive to biologic DMARDs or tsDMARDs (ie, no other biologic DMARDs or tsDMARDs qualifying as index drugs were used before the index date. The adalimumab and etanercept cohorts had the largest percentages of naive patients: $95.0 \%$ and $91.3 \%$, respectively.

Of those patients with previous biologic or tsDMARD use, the mean number of treatments in the baseline period ranged from 1.1 to 1.4. Previous initiation of the index drug, corticosteroid use ranged from $58.0 \%$ to $81.1 \%$, and methotrexate use ranged from $24.6 \%$ to $48.8 \%$ among all cohorts. Pre-index corticosteroid use was highest for patients indexing on tofacitinib, and methotrexate use was highest among patients indexing on infliximab. Use of both was lowest among patients indexing on ustekinumab.

\section{TREATMENT PATTERNS OVER 12 MONTHS OF FOLLOW-UP}

One-year persistence on index drug, using a 60-day gap, ranged from $31.5 \%$ among patients who indexed on ustekinumab to $59.3 \%$ among patients who indexed on golimumab-SC (Table 2). The mean duration of persistence ranged from 202 days (ustekinumab) to 276 days (golimumab-SC; Table 2 and Supplementary Figure 2, available in online article). Results from sensitivity analyses using 45and 90-day allowable gaps are shown in Table 2.

Across index drugs, $35.5 \%$ (infliximab) to $55.6 \%$ (certolizumab) of patients with PsA discontinued index therapy (gap 90+ days) with average time to discontinuation ranging from 110 days to 148 days (Table 2). Among those who discontinued therapy, $28.2 \%$ (ustekinumab) to $56.5 \%$ (golimumab-SC) switched to a different biologic or tsDMARD during follow-up. The subsequent biologic or tsDMARD that patients switched to differed by index drug. Between $8.7 \%$ (golimumab-SC) and $32.4 \%$ (ustekinumab) reinitiated the index drug after a gap of $90+$ days.

The mean PDC for the index drug during the 12-month follow-up ranged from 0.51 to 0.69 (Table 2). Patients initiating certolizumab and ustekinumab had the lowest mean PDC (0.51 and 0.53, respectively), whereas patients initiating infliximab and golimumab-IV had the highest mean PDC (0.68 and 0.69, respectively). The proportion of patients highly adherent to the index drug (ie, PDC $\geq 0.80$ ) during the 12 -month follow-up ranged from $25 \%$ to $48 \%$. 


\section{TABLE 2 Treatment Patterns Measured During the 12 Months of Follow-up}

\begin{tabular}{|c|c|c|c|c|c|c|c|c|c|c|}
\hline & $\begin{array}{c}\text { ADA } \\
n=2,481\end{array}$ & $\begin{array}{l}\text { CERT } \\
\mathrm{n}=71\end{array}$ & $\begin{array}{c}\text { ETA } \\
n=1,200\end{array}$ & $\begin{array}{c}\text { GOL-IV } \\
\mathrm{n}=54\end{array}$ & $\begin{array}{c}\text { GOL-SC } \\
\mathrm{n}=59\end{array}$ & $\begin{array}{c}\text { INF } \\
n=203\end{array}$ & $\begin{array}{c}\text { SEC } \\
\mathrm{n}=367\end{array}$ & $\begin{array}{c}\text { UST } \\
n=362\end{array}$ & $\begin{array}{c}\text { APR } \\
n=1,705\end{array}$ & $\begin{array}{c}\text { TOF } \\
\mathbf{n}=37\end{array}$ \\
\hline $\begin{array}{l}\text { Persistent on index drug, } \\
<60 \text {-day gap, } \mathrm{n}(\%)\end{array}$ & $\begin{array}{l}1,195 \\
(48.2)\end{array}$ & $\begin{array}{c}65 \\
(38.0)\end{array}$ & $\begin{array}{c}526 \\
(44.0)\end{array}$ & $\begin{array}{c}27 \\
(50.0)\end{array}$ & $\begin{array}{c}35 \\
(59.3)\end{array}$ & $\begin{array}{l}115 \\
(56.7)\end{array}$ & $\begin{array}{c}206 \\
(56.1)\end{array}$ & $\begin{array}{c}114 \\
(31.5)\end{array}$ & $\begin{array}{c}759 \\
(45.5)\end{array}$ & $\begin{array}{c}20 \\
(54.1)\end{array}$ \\
\hline $\begin{array}{l}\text { Days persistent on index drug, } \\
\text { mean (SD) }\end{array}$ & $\begin{array}{l}250 \\
(125)\end{array}$ & $\begin{array}{c}214 \\
(132)\end{array}$ & $\begin{array}{l}235 \\
(130)\end{array}$ & $\begin{array}{c}271 \\
(115)\end{array}$ & $\begin{array}{l}276 \\
(117)\end{array}$ & $\begin{array}{l}266 \\
(128)\end{array}$ & $\begin{array}{l}266 \\
(125)\end{array}$ & $\begin{array}{c}202 \\
(132)\end{array}$ & $\begin{array}{c}232 \\
(135)\end{array}$ & $\begin{array}{l}253 \\
(134)\end{array}$ \\
\hline $\begin{array}{l}\text { Persistent on index drug - } \\
\text { sensitivity, <45-day gap, } \mathrm{n}(\%)\end{array}$ & $\begin{array}{l}1,086 \\
(43.8)\end{array}$ & $\begin{array}{c}55 \\
(32.2)\end{array}$ & $\begin{array}{c}468 \\
(39.0)\end{array}$ & $\begin{array}{c}24 \\
(44.4)\end{array}$ & $\begin{array}{c}29 \\
(49.2)\end{array}$ & $\begin{array}{c}108 \\
(53.2)\end{array}$ & $\begin{array}{c}188 \\
(51.2)\end{array}$ & $\begin{array}{c}81 \\
(22.4)\end{array}$ & $\begin{array}{c}690 \\
(40.5)\end{array}$ & $\begin{array}{c}19 \\
(51.4)\end{array}$ \\
\hline $\begin{array}{l}\text { Persistent on index drug - } \\
\text { sensitivity, <90-day gap, } \mathrm{n}(\%)\end{array}$ & $\begin{array}{l}1,337 \\
(53.9)\end{array}$ & $\begin{array}{c}76 \\
(44.4)\end{array}$ & $\begin{array}{c}609 \\
(50.8)\end{array}$ & $\begin{array}{c}30 \\
(55.6)\end{array}$ & $\begin{array}{c}36 \\
(61.0)\end{array}$ & $\begin{array}{c}131 \\
(64.5)\end{array}$ & $\begin{array}{l}229 \\
(62.4)\end{array}$ & $\begin{array}{c}192 \\
(53.0)\end{array}$ & $\begin{array}{c}872 \\
(51.1)\end{array}$ & $\begin{array}{c}21 \\
(56.8)\end{array}$ \\
\hline $\begin{array}{l}\text { Discontinue, } \geq 90 \text {-day gap, } \\
\text { n (\%) }\end{array}$ & $\begin{array}{l}1,144 \\
(46.1)\end{array}$ & $\begin{array}{c}95 \\
(55.6)\end{array}$ & $\begin{array}{c}591 \\
(49.3)\end{array}$ & $\begin{array}{c}24 \\
(44.4)\end{array}$ & $\begin{array}{c}23 \\
(39.0)\end{array}$ & $\begin{array}{c}72 \\
(35.5)\end{array}$ & $\begin{array}{l}138 \\
(37.6)\end{array}$ & $\begin{array}{l}170 \\
(47.0)\end{array}$ & $\begin{array}{c}833 \\
(48.9)\end{array}$ & $\begin{array}{c}16 \\
(43.2)\end{array}$ \\
\hline $\begin{array}{l}\text { Days to discontinuation } \\
\text { among those who } \\
\text { discontinue, mean (SD) }\end{array}$ & $\begin{array}{l}133 \\
(72)\end{array}$ & $\begin{array}{l}110 \\
(65)\end{array}$ & $\begin{array}{l}122 \\
(70)\end{array}$ & $\begin{array}{l}148 \\
(93)\end{array}$ & $\begin{array}{l}139 \\
(65)\end{array}$ & $\begin{array}{l}120 \\
(77)\end{array}$ & $\begin{array}{l}127 \\
(75)\end{array}$ & $\begin{array}{c}119.0 \\
(79)\end{array}$ & $\begin{array}{l}112 \\
(74)\end{array}$ & $\begin{array}{c}123.0 \\
(82)\end{array}$ \\
\hline $\begin{array}{l}\text { Switching to another biologic } \\
\text { or tsDMARD, } n\end{array}$ & 519 & 49 & 285 & 9 & 13 & 32 & 52 & 48 & 290 & 7 \\
\hline$\%$ of all patients & 20.9 & 28.7 & 23.8 & 16.7 & 22.0 & 15.8 & 14.2 & 13.3 & 17.0 & 18.9 \\
\hline $\begin{array}{l}\text { Most common drug switched } \\
\text { to among those who switch } \\
\text { (\%) }\end{array}$ & $\begin{array}{c}\text { ETA } \\
(33.7)\end{array}$ & $\begin{array}{l}\text { SEC } \\
(20.4)\end{array}$ & $\begin{array}{c}\text { ADA } \\
(53.7)\end{array}$ & $\begin{array}{l}\text { ABAT-IV } \\
(33.3)\end{array}$ & $\begin{array}{l}\text { SEC } \\
(23.1)\end{array}$ & $\begin{array}{c}\text { APR } \\
(31.3)\end{array}$ & $\begin{array}{l}\text { IXE } \\
(26.9)\end{array}$ & $\begin{array}{l}\text { SEC } \\
(43.8)\end{array}$ & $\begin{array}{c}\text { ADA } \\
(36.2)\end{array}$ & $\begin{array}{l}\text { ETA, ADA } \\
\quad(28.6)\end{array}$ \\
\hline$\%$ of discontinuers & 45.4 & 51.6 & 48.2 & 37.5 & 56.5 & 44.4 & 37.7 & 28.2 & 34.8 & 43.8 \\
\hline Reinitiation of index therapy, $n$ & 127 & 9 & 86 & 4 & 2 & 9 & 21 & 55 & 117 & 2 \\
\hline$\%$ of all patients & 5.1 & 5.3 & 7.2 & 7.4 & 3.4 & 4.4 & 5.7 & 15.2 & 6.9 & 5.4 \\
\hline$\%$ of discontinuers & 11.1 & 9.5 & 14.6 & 16.7 & 8.7 & 12.5 & 15.2 & 32.4 & 14.0 & 12.5 \\
\hline $\begin{array}{l}\text { PDC of index drug over } \\
\text { 12-month follow-up, } \\
\text { mean (SD) }\end{array}$ & $\begin{array}{l}0.61 \\
(0.29)\end{array}$ & $\begin{array}{l}0.51 \\
(0.30)\end{array}$ & $\begin{array}{l}0.58 \\
(0.30)\end{array}$ & $\begin{array}{l}0.69 \\
(0.31)\end{array}$ & $\begin{array}{l}0.66 \\
(0.27)\end{array}$ & $\begin{array}{l}0.68 \\
(0.31)\end{array}$ & $\begin{array}{c}0.64 \\
(0.29)\end{array}$ & $\begin{array}{c}0.53 \\
(0.27)\end{array}$ & $\begin{array}{l}0.56 \\
(0.31)\end{array}$ & $\begin{array}{c}0.63 \\
(0.31)\end{array}$ \\
\hline $\begin{array}{l}\text { Highly adherent, PDC } \geq 0.80 \text {, } \\
\mathrm{n}(\%)\end{array}$ & $\begin{array}{c}975 \\
(39.3)\end{array}$ & $\begin{array}{c}42 \\
(24.6)\end{array}$ & $\begin{array}{c}408 \\
(34.0)\end{array}$ & $\begin{array}{c}26 \\
(48.2)\end{array}$ & $\begin{array}{c}25 \\
(42.4)\end{array}$ & $\begin{array}{c}96 \\
(47.3) \\
\end{array}$ & $\begin{array}{c}164 \\
(44.7)\end{array}$ & $\begin{array}{c}76 \\
(21.0)\end{array}$ & $\begin{array}{c}570 \\
(33.4)\end{array}$ & $\begin{array}{c}17 \\
(46.0)\end{array}$ \\
\hline $\begin{array}{l}\text { PDC of index drug before } \\
\text { discontinuation, mean (SD) }\end{array}$ & $\begin{array}{c}0.88 \\
(0.12) \\
\end{array}$ & $\begin{array}{c}0.86 \\
(0.14) \\
\end{array}$ & $\begin{array}{c}0.87 \\
(0.13) \\
\end{array}$ & $\begin{array}{c}0.93 \\
(0.12)\end{array}$ & $\begin{array}{c}0.89 \\
(0.10)\end{array}$ & $\begin{array}{c}0.90 \\
(0.13)\end{array}$ & $\begin{array}{c}0.85 \\
(0.12)\end{array}$ & $\begin{array}{c}0.80 \\
(0.20)\end{array}$ & $\begin{array}{c}0.87 \\
(0.13)\end{array}$ & $\begin{array}{c}0.88 \\
(0.10)\end{array}$ \\
\hline $\begin{array}{l}\text { MPR of index drug over } \\
\text { 12-month follow-up, mean (SD) }\end{array}$ & $\begin{array}{c}0.63 \\
(0.30)\end{array}$ & $\begin{array}{c}0.52 \\
(0.30)\end{array}$ & $\begin{array}{c}0.59 \\
(0.31)\end{array}$ & $\begin{array}{c}0.69 \\
(0.31)\end{array}$ & $\begin{array}{c}0.69 \\
(0.28)\end{array}$ & $\begin{array}{c}0.69 \\
(0.31)\end{array}$ & $\begin{array}{c}0.66 \\
(0.30)\end{array}$ & $\begin{array}{c}0.55 \\
(0.28)\end{array}$ & $\begin{array}{c}0.58 \\
(0.32)\end{array}$ & $\begin{array}{c}0.65 \\
(0.32)\end{array}$ \\
\hline $\begin{array}{l}\text { Highly adherent, MPR } \geq 0.80 \text {, } \\
\mathrm{n}(\%)\end{array}$ & $\begin{array}{l}1,051 \\
(42.4)\end{array}$ & $\begin{array}{c}43 \\
(25.2)\end{array}$ & $\begin{array}{l}449 \\
(37.4)\end{array}$ & $\begin{array}{c}26 \\
(48.2)\end{array}$ & $\begin{array}{c}29 \\
(49.2)\end{array}$ & $\begin{array}{c}96 \\
(47.3)\end{array}$ & $\begin{array}{c}181 \\
(49.3)\end{array}$ & $\begin{array}{c}85 \\
(23.5)\end{array}$ & $\begin{array}{c}648 \\
(38.0)\end{array}$ & $\begin{array}{c}19 \\
(51.4)\end{array}$ \\
\hline $\begin{array}{l}\text { MPR of index drug before } \\
\text { discontinuation, mean (SD) }\end{array}$ & $\begin{array}{c}0.90 \\
(0.12)\end{array}$ & $\begin{array}{l}0.87 \\
(0.13)\end{array}$ & $\begin{array}{c}0.89 \\
(0.13)\end{array}$ & $\begin{array}{c}0.93 \\
(0.12)\end{array}$ & $\begin{array}{c}0.91 \\
(0.09)\end{array}$ & $\begin{array}{c}0.90 \\
(0.13)\end{array}$ & $\begin{array}{c}0.87 \\
(0.13)\end{array}$ & $\begin{array}{l}0.80 \\
(0.20)\end{array}$ & $\begin{array}{c}0.88 \\
(0.13)\end{array}$ & $\begin{array}{l}0.90 \\
(0.10)\end{array}$ \\
\hline
\end{tabular}

Adherence to the index drug before discontinuation was high, with the mean PDC before discontinuation ranging from 0.80 to 0.93 across the index drug cohorts. Results from MPR sensitivity analysis were consistent with PDC and are included in Table 2.

Concurrent with index drug treatment, 48.3\%-68.0\% had at least 1 claim for corticosteroids, and 15.7\%-42.6\% had at least 1 claim for methotrexate (Table 2). Concomitant use of sulfasalazine or leflunomide was below $10.2 \%$ in any cohort, and concomitant use of azathioprine or cyclosporine was below $2 \%$.

\section{DOSING}

With 1 exception (secukinumab), dosing measured in realworld data was consistent with prescribing information for all biologics and tsDMARDs included in this analysis (Table 3 ). 
TABLE 2 Treatment Patterns Measured During the 12 Months of Follow-up (continued)

\begin{tabular}{|c|c|c|c|c|c|c|c|c|c|c|}
\hline & $\begin{array}{c}\text { ADA } \\
n=2,481\end{array}$ & $\begin{array}{l}\text { CERT } \\
\mathrm{n}=71\end{array}$ & $\begin{array}{c}\text { ETA } \\
n=1,200\end{array}$ & $\begin{array}{c}\text { GOL-IV } \\
n=54\end{array}$ & $\begin{array}{c}\text { GOL-SC } \\
n=59\end{array}$ & $\begin{array}{c}\text { INF } \\
n=203\end{array}$ & $\begin{array}{c}\text { SEC } \\
n=367\end{array}$ & $\begin{array}{c}\text { UST } \\
n=362\end{array}$ & $\begin{array}{c}\text { APR } \\
\mathrm{n}=1,705\end{array}$ & $\begin{array}{c}\text { TOF } \\
\mathrm{n}=37\end{array}$ \\
\hline \multicolumn{11}{|c|}{ Concomitant medication use, $\mathrm{n}(\%)$} \\
\hline $\begin{array}{l}\text { Corticosteroids } \\
\text { (oral or injectable) }\end{array}$ & $\begin{array}{l}1,197 \\
(48.3) \\
\end{array}$ & $\begin{array}{c}101 \\
(59.1)\end{array}$ & $\begin{array}{c}612 \\
(51.0) \\
\end{array}$ & $\begin{array}{c}34 \\
(63.0) \\
\end{array}$ & $\begin{array}{c}40 \\
(67.8) \\
\end{array}$ & $\begin{array}{c}138 \\
(68.0) \\
\end{array}$ & $\begin{array}{c}206 \\
(56.1) \\
\end{array}$ & $\begin{array}{c}177 \\
(48.9) \\
\end{array}$ & $\begin{array}{c}841 \\
(49.3) \\
\end{array}$ & $\begin{array}{c}23 \\
(62.2) \\
\end{array}$ \\
\hline Methotrexate & $\begin{array}{c}726 \\
(29.3)\end{array}$ & $\begin{array}{c}53 \\
(31.0)\end{array}$ & $\begin{array}{c}301 \\
(25.1)\end{array}$ & $\begin{array}{c}23 \\
(42.6)\end{array}$ & $\begin{array}{c}22 \\
(37.3)\end{array}$ & $\begin{array}{c}84 \\
(41.4)\end{array}$ & $\begin{array}{c}89 \\
(24.3)\end{array}$ & $\begin{array}{c}62 \\
(17.1)\end{array}$ & $\begin{array}{c}267 \\
(15.7)\end{array}$ & $\begin{array}{c}13 \\
(35.1)\end{array}$ \\
\hline Leflunomide & $\begin{array}{c}77 \\
(3.1)\end{array}$ & $\begin{array}{c}12 \\
(7.0)\end{array}$ & $\begin{array}{c}48 \\
(4.0)\end{array}$ & $\begin{array}{c}5 \\
(9.3) \\
\end{array}$ & $\begin{array}{c}5 \\
(8.5) \\
\end{array}$ & $\begin{array}{l}10 \\
(4.9)\end{array}$ & $\begin{array}{c}15 \\
(4.1)\end{array}$ & $\begin{array}{c}13 \\
(3.6)\end{array}$ & $\begin{array}{c}50 \\
(2.9)\end{array}$ & $\begin{array}{c}3 \\
(8.1) \\
\end{array}$ \\
\hline Sulfasalazine & $\begin{array}{l}125 \\
(5.0)\end{array}$ & $\begin{array}{l}11 \\
(6.4)\end{array}$ & $\begin{array}{c}72 \\
(6.0)\end{array}$ & $\begin{array}{c}4 \\
(7.4)\end{array}$ & $\begin{array}{c}6 \\
(10.2)\end{array}$ & $\begin{array}{l}15 \\
(7.4)\end{array}$ & $\begin{array}{c}22 \\
(6.0)\end{array}$ & $\begin{array}{c}14 \\
(3.9)\end{array}$ & $\begin{array}{c}79 \\
(4.6)\end{array}$ & $\begin{array}{c}2 \\
(5.4)\end{array}$ \\
\hline Cyclosporine & $\begin{array}{l}10 \\
(0.4)\end{array}$ & $\begin{array}{c}2 \\
(1.2)\end{array}$ & $\begin{array}{c}4 \\
(0.3) \\
\end{array}$ & $\begin{array}{c}0 \\
(0.0)\end{array}$ & $\begin{array}{c}0 \\
(0.0)\end{array}$ & $\begin{array}{c}2 \\
(1.0) \\
\end{array}$ & $\begin{array}{c}2 \\
(0.5)\end{array}$ & $\begin{array}{c}5 \\
(1.4) \\
\end{array}$ & $\begin{array}{c}3 \\
(0.2) \\
\end{array}$ & $\begin{array}{c}0 \\
(0.0)\end{array}$ \\
\hline Azathioprine & $\begin{array}{c}8 \\
(0.3)\end{array}$ & $\begin{array}{c}1 \\
(0.6)\end{array}$ & $\begin{array}{c}5 \\
(0.4)\end{array}$ & $\begin{array}{c}0 \\
(0.0)\end{array}$ & $\begin{array}{c}1 \\
(1.7)\end{array}$ & $\begin{array}{c}3 \\
(1.5)\end{array}$ & $\begin{array}{c}1 \\
(0.3)\end{array}$ & $\begin{array}{c}3 \\
(0.8)\end{array}$ & $\begin{array}{c}5 \\
(0.3)\end{array}$ & $\begin{array}{c}0 \\
(0.0)\end{array}$ \\
\hline
\end{tabular}

$A D A=$ adalimumab; $A P R=$ apremilast; $C E R T=$ certolizumab; $E T A=$ etanercept; $G O L-I V=$ golimumab intravenous; GOL-SC=golimumab subcutaneous; INF=infliximab; $I X E=$ ixekizumab; $M P R=$ medication possession ratio; $P D C=$ proportion of days covered; $S E C=$ secukinumab; $t$ DMARDs=targeted synthetic disease-modifying antirheumatic drugs; TOF=tofacitinib; UST = ustekinumab.

Secukinumb PsA patients with comorbid PsO received a median weekly induction dosage of $150 \mathrm{mg}$, rather than the recommended dosage of $300 \mathrm{mg}$ for patients with moderate to severe PsO. ${ }^{23}$ Meanwhile, PsA patients without comorbid $\mathrm{PsO}$ had a median monthly dose of $300 \mathrm{mg}$, at the high end of the 150-300 mg range for maintenance dosing. Further, while induction dosing is optional for PsA patients without comorbid $\mathrm{PsO}$, the median induction dosing was $150 \mathrm{mg}$ weekly, indicating that most PsA-only patients in this study had dosing consistent with an induction period.

\section{HEALTH CARE COSTS}

The mean (SD) all-cause PPPM health care costs during the 12-month follow-up period ranged from $\$ 4,353$ $(\$ 5,487)$ for apremilast to $\$ 6,640(\$ 5,191)$ for ustekinumab (Supplementary Table 1, available in online article) The mean (SD) PsA-related PPPM health care costs ranged from $\$ 2,647(\$ 1,583)$ for the apremilast cohort to $\$ 5,159(\$ 3,155)$ for the ustekinumab cohort (Supplementary Table 1). Mean index drug costs PPPM ranged from $\$ 1,874$ (SD \$1,126) in the apremilast cohort to $\$ 4,514$ (SD $\$ 3,163$ ) in the ustekinumab cohort (Figure 1). PsA index drug costs comprised the majority (69\%-88\%) of total PsA-related health care costs, but only $2.9 \%$ to $6.4 \%$ of index drug costs were patient outof-pocket expenses (Supplementary Table 1).

After applying ICER drug-specific discounts, the adjusted monthly index drug costs decreased to between $\$ 1,461$ (apremilast) and $\$ 3,295$ (ustekinumab), which reduced total
PsA-related health care costs in all index cohorts, with a range of $\$ 2,044$ (apremilast) to $\$ 3,772$ (ustekinumab; Table 4).

Index drug costs may also be affected by adherence rates. Patients who are more adherent to treatment have, by definition, higher drug costs than those who are not adherent. As such, index drug costs were adjusted by both ICER discount and adherence rate to compare costs, assuming that all patients were adherent to their treatment. After ICER-adherence adjustment, index drug costs PPPM ranged from $\$ 2,361$ (\$257) for apremilast to $\$ 6,528(\$ 4,152)$ for ustekinumab.

\section{Discussion}

In this study, we examined treatment patterns across a large group of PsA-indicated biologics and tsDMARDs. Across all treatment cohorts, $31 \%$ to $59 \%$ of patients remained persistent on index therapy, and $35 \%$ to $56 \%$ discontinued the index therapy. Among those who discontinued, $9 \%$ to $32 \%$ restarted their index drug, whereas $28 \%$ to $57 \%$ switched to a different biologic or tsDMARD. Before discontinuation, mean PDC of the index drug ranged from 0.80 to 0.93 , whereas during the full 12-month follow-up, mean PDC of the index drug ranged from 0.51 to 0.69 . During the 12-month follow-up, $48 \%$ to $68 \%$ of PsA patients had at least 1 claim for corticosteroids, which suggests continuing unmet medical need despite the high PDC before discontinuation.

Findings on persistence, switching, and discontinuation rates were consistent with previously published studies 


\section{TABLE 3}

\section{Dosing Measured in Claims and Compared with Prescribing Information}

\begin{tabular}{|c|c|c|}
\hline Dosing among eligible patients ${ }^{a}$ & $\begin{array}{l}\text { Calculated } \\
\text { dose in claims } \\
(\mathrm{mg})\end{array}$ & $\begin{array}{c}\text { Expected } \\
\text { dose based on } \\
\text { prescribing } \\
\text { information } \\
\text { (mg) }\end{array}$ \\
\hline \multicolumn{3}{|c|}{ Dose for index therapies with a split dosing phase } \\
\hline \multicolumn{3}{|l|}{ Certolizumab $(n=154)$} \\
\hline \multicolumn{3}{|l|}{ Induction phase } \\
\hline Median biweekly dose in mg & 400 & 400 \\
\hline \multicolumn{3}{|l|}{ Maintenance phase } \\
\hline Median biweekly dose in mg & 200 & 200 \\
\hline \multicolumn{3}{|c|}{ Secukinumab, without comorbid PsO $(n=79)$} \\
\hline \multicolumn{3}{|l|}{ Induction phase } \\
\hline Median weekly dose in $\mathrm{mg}$ & 150 & 150 \\
\hline \multicolumn{3}{|l|}{ Maintenance phase } \\
\hline Median monthly dose in $\mathrm{mg}$ & 300 & $\begin{array}{c}150 \text { or can } \\
\text { increase to } 300 \\
\text { if patient still } \\
\text { has active PsA }\end{array}$ \\
\hline
\end{tabular}

Secukinumab, with comorbid PsO $(n=260)$

Induction phase

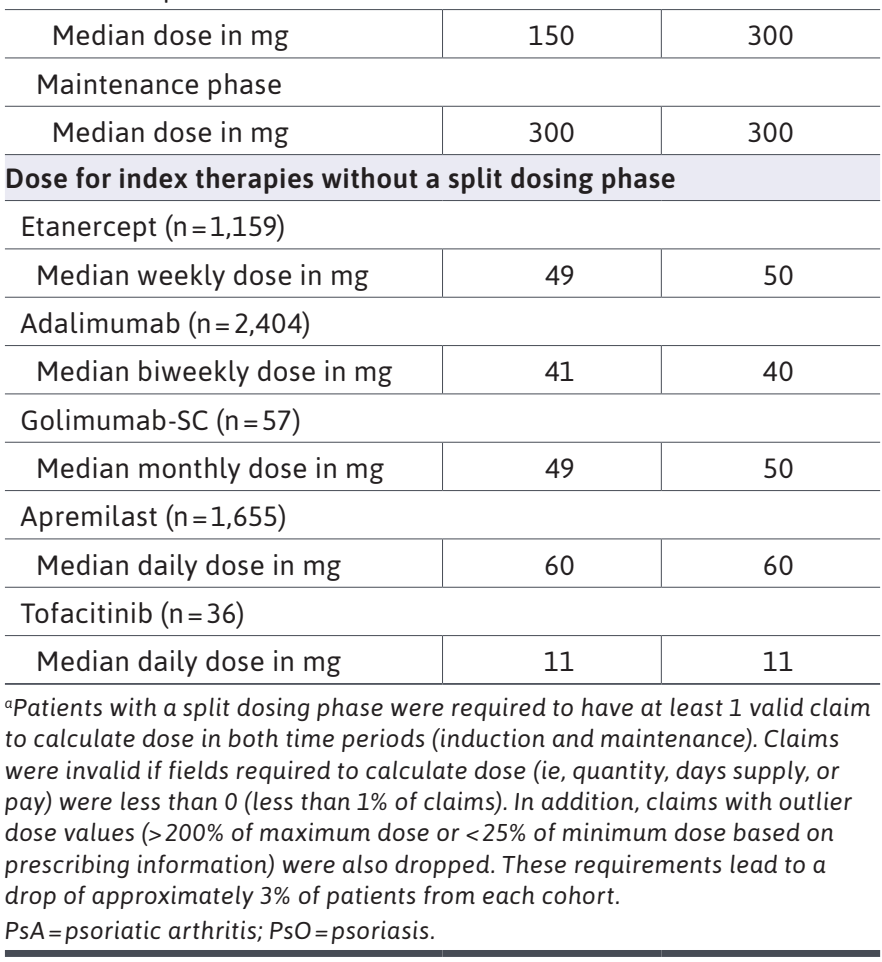

on tsDMARD and biologic use in psoriatic disease, which reported persistence rates of 32\%-61\%, ${ }^{15,24}$ discontinuations rates of $35 \%-56 \%,{ }^{13-16}$ index drug restart rates of $5 \%-15 \%,{ }^{14,15}$ switching rates of $2 \%-28 \%,{ }^{14-16,24}$ and adherence rates of 0.45-0.6711-13 over 12 months of treatment. Previous studies also found that patients using certolizumab pegol had low persistence and adherence rates, ${ }^{13,15}$ and another study found that among Medicare patients initiating a select biologic DMARD (adalimumab, etanercept, infliximab, and ustekinumab), the rate of restart after discontinuation was highest among ustekinumab initiators, ${ }^{14}$ which was consistent with the findings of our study.

Because the first study drug observed in the selection period was flagged as the index drug, the majority (63\%95\%) of patients in each index drug cohort were biologic- or tsDMARD-naive (no other biologic or tsDMARD therapies in the 12 months before index therapy initiation). The percentage of PsA patients with comorbid PsO ranged from $46 \%$ to $78 \%$, depending on the index drug. The percentage of patients with comorbid PsO tended to be higher (54\%-78\%) for patients on medications approved to treat both PsA and $\mathrm{PsO}$ and lower (46\%-54\%) for patients on medications only approved to treat PsA. This is consistent with previous studies that report that $50 \%$ of newly diagnosed PsA patients and $55 \%$ to $74 \%$ of biologic-treated PsA patients had a recent $\mathrm{PsO}$ diagnosis code. ${ }^{10,13}$ Percentages of patients with other reported comorbidities were also in line with those reported previously. ${ }^{13,25}$

Observed dosing before discontinuation was generally consistent with prescribing recommendations for PsA for all study drugs, with the exception of secukinumab. In this study, the observed median secukinumab induction dose was $150 \mathrm{mg}$ per week and the observed median maintenance dose was $300 \mathrm{mg}$ per week for patients with and without comorbid PsO. These data suggest that prescribers may not be altering induction dosing based on comorbid PsO status and that they are also prescribing the higher dose during maintenance, regardless of comorbid PsO status.

In this study, apremilast initiators had the lowest PPPM drug and health care costs. This is consistent with a previous study by Feldman et al that compared costs among patients initiating biologics with those initiating apremilast and found that monthly all-cause health care costs for apremilast users were $\$ 875$ lower than for biologic users. ${ }^{24}$ Average unadjusted PPPM drug costs for apremilast initiators in our study were slightly lower than those adjusted costs reported in the previous study (\$1,874 vs $\$ 2,581)$, and average PPPM drug costs for biologics in our study ranged from $\$ 2,519$ to $\$ 4,514$, which was similar to the average PPPM biologics cost of $\$ 3,264$. Consistent with the Feldman et al study, apremilast initiators in our study did not exhibit 


\section{FIGURE 1 Average PsA-Related Health Care Costs Reported PPPM and Stratified by Index Drug}

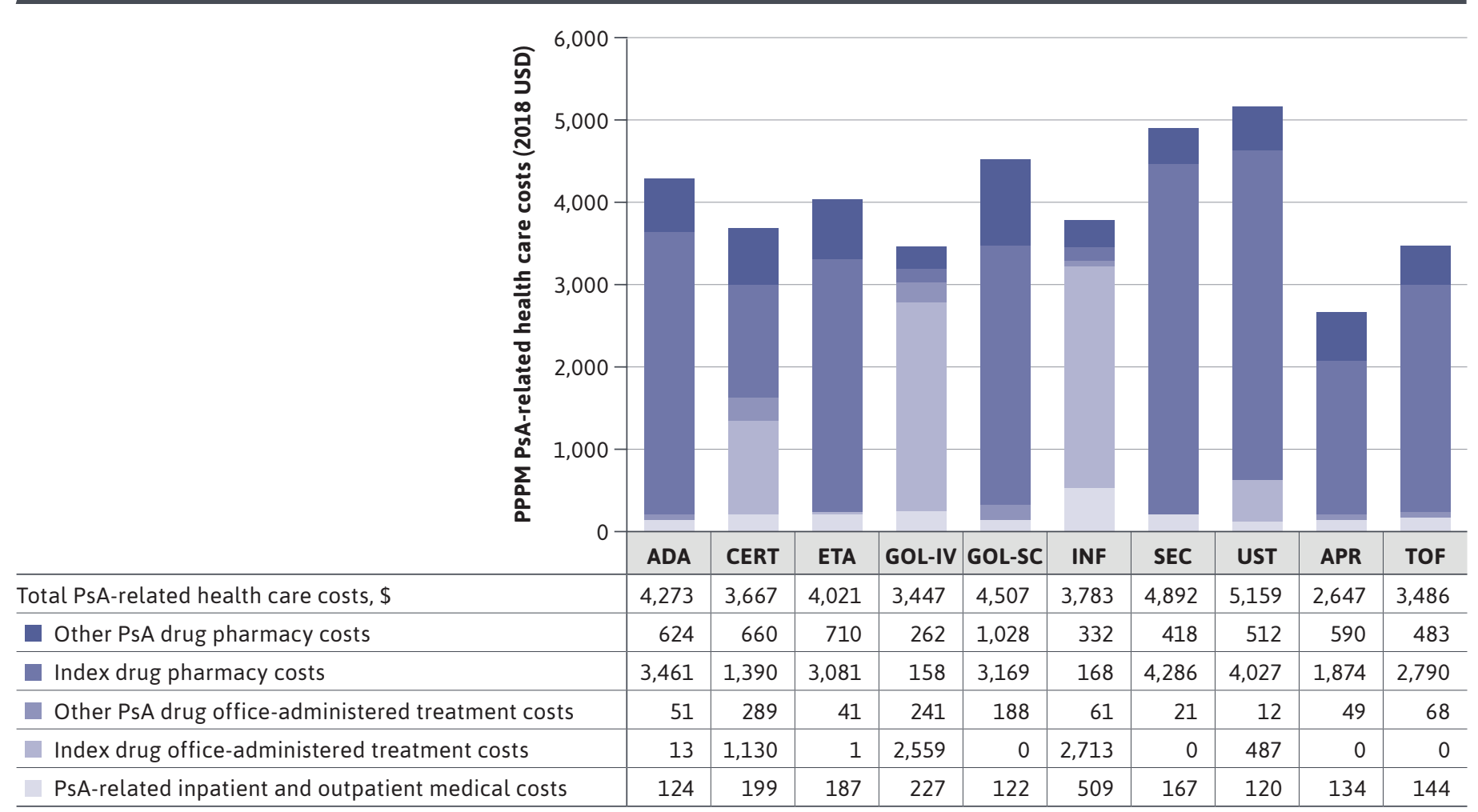

$A D A=$ adalimumab; $A P R=$ apremilast; $C E R T=$ certolizumab; $E T A=$ etanercept; $G O L-I V=$ golimumab intravenous; $G O L-S C=$ golimumab subcutaneous; $I N F=$ infliximab; PPPM = per patient per month; PSA=psoriatic arthritis; SEC=secukinumab; TOF=tofacitinib; UST=ustekinumab.

higher or lower persistence or adherence to index drug relative to those initiating biologics.

PsA index drug costs comprised the majority (69\%-88\%) of total PsA-related health care costs, but patients paid just $6.4 \%$ of these index drug costs or less. Adjusting the index drug costs by ICER discounts and adherence illustrated the effect of applied discounts on index treatment costs and provide a more realistic estimation of real-world index costs for adherent patients.

\section{LIMITATIONS}

This study is subject to limitations. Several factors that influence treatment patterns, such as PsA disease duration and disease severity, are not available in claims data and therefore are not accounted for in this analysis. Furthermore, these datasets are subject to misclassification (ie, miscoding or undercoding) and coding limitations, which may have introduced bias or measurement error. The Early View database may have missed $1 \%-3 \%$ of pharmacy claims because of the shorter adjudication period. However, these biases are expected to be similar across treatment groups.

This study was limited to individuals with commercial or private Medicare supplemental coverage. Results of this analysis may not be generalizable to patients with other or no health insurance coverage. Treatment pattern analyses were built on the assumption that patients used their medications as prescribed and dispensed, but that does not mean the patient actually used the therapy.

In addition, the study cohort was a mix of biologicor tsDMARD-naive and experienced patients, with some patients initiating index treatment as first line and others as later line. It is possible that some treatments were more likely to be used as first line and other more likely to be used as later line. Treatment adherence, switching, and discontinuation are often associated with line of therapy. We may have underestimated the line of therapy, given the relatively short baseline period. Furthermore, confounding by indication more broadly makes comparison among 


\section{TABLE 4 ICER-Adjusted PsA-Related Costs}

\begin{tabular}{|c|c|c|c|c|c|c|c|}
\hline & $\begin{array}{c}\text { ADA } \\
n=2,481\end{array}$ & $\begin{array}{c}\text { CERT } \\
\mathrm{n}=171\end{array}$ & $\begin{array}{c}\text { ETA } \\
n=1,200\end{array}$ & $\begin{array}{c}\text { INF } \\
n=203\end{array}$ & $\begin{array}{c}\text { SEC } \\
n=367\end{array}$ & $\begin{array}{c}\text { UST } \\
n=362\end{array}$ & $\begin{array}{c}\text { APR } \\
\mathrm{n}=1,705\end{array}$ \\
\hline Index drug discount (\%) & 31 & 36 & 31 & 22 & 38 & 27 & 22 \\
\hline ICER-adjusted index drug costs (mean), \$ & 2,397 & 1,612 & 2,127 & 2,247 & 2,657 & 3,295 & 1,461 \\
\hline ICER adherence-adjusted index drug costs, \$ & 3,640 & 3,346 & 3,439 & 3,473 & 4,338 & 6,528 & 2,361 \\
\hline Other PsA-related drug discounts ${ }^{a}(\%)$ & 30 & 25 & 29 & 25 & 30 & 32 & 30 \\
\hline ICER-adjusted other PsA-related drug costs (mean), \$ & 473 & 709 & 535 & 293 & 308 & 356 & 448 \\
\hline Total PsA-related health care costs discounts (\%) & 30 & 31 & 29 & 19 & 36 & 27 & 23 \\
\hline ICER-adjusted total PsA-related health care costs (mean), \$ & 2,994 & 2,520 & 2,849 & 3,050 & 3,132 & 3,772 & 2,044 \\
\hline
\end{tabular}

${ }^{a}$ Calculated from the individual drug discount factors applied to actual cost and utilization data from nonindex drug treatments.

$A D A=$ adalimumab; $A P R=$ apremilast CERT = certolizumab; $E T A=$ etanercept; ICER=Institute for Clinical and Economic Review; INF = infliximab; PsA=psoriatic arthritis; SEC = secukinumab; UST=ustekinumab.

therapies challenging, particularly as many of the reasons for therapy selection are unmeasured.

Health care costs, including index drug costs, were measured over a 12-month follow-up for patients initiating an index drug, which may have differed for patients who had continued the drug over a longer period of time.

Another limitation is that claims data may not accurately represent real drug transaction prices, since they may not capture discounts, rebates, or other patient assistant programs. As such, we attempted to overcome this issue by applying discounts from a comprehensive analysis performed by ICER. However, underestimation or overestimation may occur due to several reasons. First, some rebates and patient assistance programs may have been reflected in claims. Second, ICER discount factors were used to adjust costs for 2016 through 2018. Third, the ICER discount rates that were used were calculated for $\mathrm{PsO}$ patients (ICER PsA rates are not available), and the discount programs available to patients with PsA may be different (although unlikely, since discounts are associated with medications and not individual indications).

\section{Conclusions}

In this study, we compared drug persistence, adherence, discontinuation, switching, and reinitiation rates of both older and more recently approved biologics and tsDMARDs indicated for PsA. Our findings were consistent with previously published studies that examined treatment pattern outcomes, but they provide a more updated analysis of treatment pattern outcomes for these drugs using consistent definitions for easier comparison across treatments. In addition, ICER adherence-adjusted drug costs may provide more accurate cost estimates for these treatments, and when combined with treatment patterns, this information may help to better inform treatment and/or formulary decisions.

\section{DISCLOSURES}

This study was funded by Eli Lilly Inc., which participated in analysis and interpretation of data, drafting, reviewing, and approving the publication. All authors contributed to the development of the publication and maintained control over the final content. Murage, Malatestinic, Zhu, Atiya, Kern, Stenger, and Sprabery are employees and stockholders of Eli Lilly Inc. Princic and Park are employed by IBM
Watson Health, which received funding from Eli Lilly Inc. to conduct this study. Ogdie has received consulting fees from Amgen, AbbVie, Bristol-Myers Squibb, Celgene, Corrona, Janssen, Lilly, Novartis, and Pfizer and has also received grant support from Pfizer, Novartis, and Amgen.

Portions of these data have been presented in poster form at the virtual International Society for Pharmacoeconomics and Outcomes Research (ISPOR) 2020 and Congress of Clinical Rheumatology (CCR) West 2020 conferences.

\section{ACKNOWLEDGMENTS}

Medical writing services were provided by Jessamine Winer-Jones, PhD, of IBM Watson Health. Programming services were provided by Caroline Henriques of IBM Watson Health. These services were paid for by Eli Lilly Inc.

\section{REFERENCES}

1. Belasco J, Wei N. Psoriatic arthritis: what is happening at the joint? Rheumatol Ther. 2019;6(3):305-15.

2. Gladman DD, Antoni C, Mease P, Clegg DO, Nash P. Psoriatic arthritis: epidemiology, clinical features, course, and outcome. Ann Rheum Dis. 2005;64 (Suppl 2):ii14-17.

3. Ogdie A, Weiss P. The epidemiology of psoriatic arthritis. Rheum Dis Clin North Am. 2015;41(4):545-68. 
4. Lee S, Mendelsohn A, Sarnes E. The burden of psoriatic arthritis: a literature review from a global health systems perspective. P. T. 2010;35(12):680-89.

5. Brezinski EA, Dhillon JS, Armstrong AW. Economic burden of psoriasis in the United States: a systematic review. JAMA Dermatol. 2015;151(6):651-58.

6. Merola J, Peterson S, Dennis N, et al. PMU15 retrospective study examining health care utilization and costs for patients with psoriasis and psoriatic arthritis in the US [abstract]. Value Health. 2020;23(Suppl 1):S235. Accessed December 9, 2021. doi:10.1016/j.jval.2020.04.791

7. Singh JA, Guyatt G, Ogdie A, et al. 2018 American College of Rheumatology/ National Psoriasis Foundation Guideline for the Treatment of Psoriatic Arthritis. Arthritis Care Res. 2019;71(1):2-29.

8. Ogdie A, Coates LC, Gladman DD. Treatment guidelines in psoriatic arthritis. Rheumatology (Oxford, England). 2020;59(Suppl 1):i37-i46.

9. Haddad A, Zisman D. Comorbidities in patients with psoriatic arthritis. Rambam Maimonides Med. 2017;8(1).

10. Kaine J, Song X, Kim G, Hur P, Palmer JB. Higher incidence rates of comorbidities in patients with psoriatic arthritis compared with the general population using U.S. administrative claims data. J Manag Care Spec Pharm. 2019;25(1):122-32. doi:10.18553/ imcp.2018.17421

11. Blauvelt A, Shi N, Burge R, et al. Comparison of real-world treatment patterns among patients with psoriasis prescribed ixekizumab or secukinumab. J Am Acad Dermatol. 2020;82(4):927-35.
12. Blauvelt A, Shi N, Burge R, et al. Comparison of real-world treatment patterns among psoriasis patients treated with ixekizumab or adalimumab. Patient Prefer Adherence. 2020;14:517-27.

13. Oelke KR, Chambenoit O, Majjhoo AQ, Gray S, Higgins K, Hur P. Persistence and adherence of biologics in US patients with psoriatic arthritis: analyses from a claims database. J Comp Eff Res. 2019;8(8):607-21.

14. Doshi JA, Takeshita J, Pinto L, et al. Biologic therapy adherence, discontinuation, switching, and restarting among patients with psoriasis in the US Medicare population. J Am Acad Dermatol. 2016;74(6):1057-65.e1054.

15. Walsh JA, Adejoro O, Chastek B, Palmer JB, Hur P. Treatment patterns among patients with psoriatic arthritis treated with a biologic in the United States: descriptive analyses from an administrative claims database. J Manag Care Spec Pharm. 2018;24(7):623-31. doi:10.18553/imcp.2018.24.7.623

16. Zhang HF, Gauthier G, Hiscock R, Curtis JR. Treatment patterns in psoriatic arthritis patients newly initiated on oral nonbiologic or biologic disease-modifying antirheumatic drugs. Arthritis Res Ther. 2014;16(4):420.

17. Blauvelt A, Papp KA, Sofen H, et al. Continuous dosing versus interrupted therapy with ixekizumab: an integrated analysis of two phase 3 trials in psoriasis. J Eur Acad Dermatol Venereol. 2017;31(6):1004-13

18. Brezinski EA, Armstrong AW. Off-label biologic regimens in psoriasis: a systematic review of efficacy and safety of dose escalation, reduction, and interrupted biologic therapy. PloS One. 2012;7(4):e33486.
19. Hur P, Kim N, Dai D, Piao OW, Zheng JZ, Yi E. Healthcare cost and utilization associated with biologic treatment patterns among patients with psoriatic arthritis: analyses from a large US claims database. Drugs Real World Outcomes. 2021;8(1):29-38.

20. Feldman SR, Tian H, Wang $X$, Germino R. Health care utilization and cost associated with biologic treatment patterns among patients with moderate to severe psoriasis: analyses from a large U.S. claims database. J Manag Care Spec Pharm. 2019;25(4):479-88. doi:10.18553/ imcp.2018.18308

21. US Bureau of Labor Statistics. Consumer Price Index. Measuring price change in the CPI: medical care. Accessed March 1, 2020. https://www.bls.gov/cpi/ factsheets/medical-care.htm

22. Banken R, Agboola F, Ellis A, et al. Targeted immunomodulators for the treatment of moderate-to-severe plaque psoriasis: effectiveness and value. Institute for Clinical and Economic Review. June 12, 2018. Accessed December 9, 2021. https://icer.org/wp-content/ uploads/2020/10/ICER Psoriasis Update Evidence Report 061218.pdf

23. Cosentyx. Package insert. Novartis Pharmaceuticals Corporation; 2015. Accessed December 9, 2021. https://www. accessdata.fda.gov/drugsatfda_docs/ label/2015/125504s000lbl.pdf

24. Feldman SR, Pelletier CL, Wilson KL, et al. Treatment patterns and costs among biologic-naive patients initiating apremilast or biologics for psoriatic arthritis. J Comp Eff Res. 2019;8(9):699-709.

25. Queiro R, Lorenzo A, Tejon P, Coto P, Pardo E. Obesity in psoriatic arthritis: comparative prevalence and associated factors. Medicine (Baltimore). 2019;98(28):e16400. 